\title{
Zukunftsperspektiven der Verhaltenstherapie
}

\author{
Michael Linden ${ }^{\mathrm{a}}$ Winfried Rief ${ }^{\mathrm{b}} \quad$ Ulrich Voderholzer ${ }^{\mathrm{c}} \quad$ Claus Vögele $^{\mathrm{d}}$ \\ ${ }^{a}$ Abteilung Verhaltenstherapie und Psychosomatik am Rehabilitationszentrum Seehof der Deutschen Rentenversicherung Bund, \\ Teltow/Berlin, Deutschland; \\ ${ }^{\mathrm{b}}$ AG Klinische Psychologie und Psychotherapie, Philipps-Universität Marburg, Marburg, Deutschland; \\ ${ }^{c}$ Abteilung für Psychosomatik, Schön Klinik Roseneck, Prien am Chiemsee, Deutschland; \\ ${ }^{\mathrm{d}}$ Institute for Health and Behaviour, INSIDE, University of Luxembourg, Walferdange, Luxembourg
}

Das 25-jährige Jubiläum unserer Zeitschrift ist ein guter Anlass dafür, einmal über die Zukunft unserer Therapierichtung nachzudenken. Wie wird sich die Verhaltenstherapie (VT) in den kommenden Jahren und Jahrzehnten weiterentwickeln? Hat sie als Behandlungskonzept und Richtlinienverfahren überhaupt eine langfristige Zukunft?

Die VT hat in Deutschland eine ca. 50- bis 60-jährige Historie. Sie hat seit ihren Anfängen mit der auf der klassischen Lerntheorie basierenden systematischen Desensibilisierung in den 1950er-Jahren inzwischen eine Reihe von theoretischen Weiterentwicklungen durchgemacht, die gerne auch als «Wellen» bezeichnet werden. In den 1960er-Jahren wurden die Sozialpsychologie in die VT integriert und Therapieverfahren wie das Assertiveness-Training oder die Interpersonelle Psychotherapie (IPT) entwickelt. Damals wurde die VT durch Prof. Brengelmann vom Max-Planck-Institut für Psychiatrie auch in Deutschland bekannt gemacht. Dann kamen Weiterentwicklungen wie die Einbeziehung von kognitiven und Attributionstheorien, der Emotionspsychologie, der Störungsorientierung (DSMIII), der Psychobiologie und Persönlichkeitspsychologie oder der Kulturpsychologie, d.h. mindestens 8 «Wellen». Mancher fragt sich, ob VT als eigenständiges Psychotherapieverfahren mittlerweile als überholt gilt, insbesondere mit Blick auf theoretische Konzepte wie allgemeine Psychotherapie, integrative Psychotherapie, modulare Psychotherapie oder störungsspezifische Psychotherapie, oder mit Blick auf Therapieschulen und -methoden wie Dialektisch-behaviorale Therapie, Schematherapie, Akzeptanz- und Commitment-Therapie, das Cognitive Behavioral Analysis System of Psychotherapy (CBASP) oder metakognitive Therapien, die zum Teil mehr «en vogue» sind und sich als teilweise verfahrensübergreifend definieren. Ist eine verfahrensorientierte Therapie überholt und einseitig, demgegenüber eine störungsorientierte, integrative und konzeptübergreifende Therapie modern oder besser?
Welche Zukunft hat also die VT? Wird sie abgelöst werden durch eine allgemeine, modulare Psychotherapie, die zwar verhaltenstherapeutische Techniken enthält, aber eben auch viele weitere Interventionen?

Wenn man über die Zukunft der VT nachdenkt, muss man auch einige gesellschaftliche und gesundheitspolitische Entwicklungen berücksichtigen:

- Obwohl die vielbeschworene Zunahme psychischer Störungen in unserer Gesellschaft aus methodischen Gründen oft bezweifelt wird, kann man eindeutig feststellen, dass die Häufigkeit der Inanspruchnahme einer Behandlung wegen psychischer Erkrankungen zugenommen hat.

- Psychotherapie erlangt derzeit im Therapiekanon eine immer größere Bedeutung. Während im Bereich der Pharmakotherapie in den letzten 10-20 Jahren kaum noch Innovationen stattgefunden haben, ist der Wissensstand über die Wirksamkeit von Psychotherapie bei psychischen Erkrankungen stark gewachsen. Die Kenntnisse bezüglich der Wirksamkeit von Psychotherapie haben in praktisch allen Leitlinien psychischer Erkrankungen, einschließlich der Schizophrenie, Eingang gefunden. Psychotherapie wird auch bei Erkrankungen empfohlen, bei denen sie bislang in der Praxis noch vergleichsweise selten zum Einsatz kommt, wie etwa bei Psychosen.

- Es besteht eine ungebremste Nachfrage nach Richtlinienpsychotherapie. Alle Richtlinienpsychotherapeuten haben Wartelisten, die je nach Region unterschiedlich lang sein können. Die Wartezeiten werden zudem auch noch durch das Antrags- und Wirtschaftlichkeitsprüfverfahren verlängert. Diese Vorlaufzeiten sind bei Verhaltenstherapeuten noch länger als bei tiefenpsychologisch orientierten Therapeuten. Aber auch für die fachärztliche Psychotherapie durch Allgemeinärzte, Psychiater oder Psychosomatiker, deren Aufgabe es unter anderem ist, bei einem akuten Problem sofort aufgesucht werden zu können, gibt es Wartezeiten von Wochen.

\section{KARGER \\ Fax +497614520714

\section{() 2015 S. Karger GmbH, Freiburg}

1016-6262/15/0251-0005\$39.50/0 
- Eine immer größer werdende Zahl von Migranten lebt in Deutschland und benötigt spezielle Formen der Psychotherapie mit besonderen Kenntnissen der Heimatsprache und Ursprungskultur.

- Der demografische Wandel schreitet voran, insbesondere durch die gestiegene Lebenserwartung. Dadurch bedingt werden die Ressourcen im Gesundheitssystem immer knapper und der ökonomische Druck steigt weiter an. Dieser Wettbewerb um die ökonomischen Ressourcen im Gesundheitssystem wird durch zunehmend teure Diagnostik- und Therapiemaßnahmen der Medizin weiter verschärft. Gleichzeitig muss das psychotherapeutische Angebot für ältere Menschen noch besser adaptiert und erweitert werden.

- Das Internet und die damit verbundenen Kommunikationsstrukturen haben die Gesellschaft in den vergangenen Jahrzehnten, insbesondere in den zurückliegenden 5-10 Jahren, stark verändert.

Welche Konsequenzen haben diese Entwicklungen für die Psychotherapie im Allgemeinen und die VT im Besonderen?

Eine Psychotherapie, die den Anforderungen dieser Entwicklungen gerecht werden kann, muss und wird sicherlich ökonomischer sein als bisher, und damit kürzer und effektiver. Darüber hinaus wird bei der Psychotherapie der Zukunft noch größerer Wert als bisher auf Evidenzbasierung gelegt werden. Schließlich wird die Nutzung neuer Medien auch im Rahmen der Psychotherapie eine größere Bedeutung erlangen. Dies bedeutet, dass ein Psychotherapieverfahren und eine psychotherapeutische Ausbildung diesen verschiedenen Organisationsformen von Psychotherapie gerecht werden müssen, d.h. sowohl der beantragungspflichtigen und umfangsmäßig kontingentierten Richtlinienpsychotherapie wie der fachärztlichen Psychotherapie, den delegationsfähigen psychosozialen Interventionen (z.B. Patientenedukation) oder der Langzeitpsychotherapie chronisch Kranker.

Andererseits gibt es Entwicklungen, deren Auswirkungen noch nicht absehbar sind. Die aktuell diskutierte Direktausbildung von Psychotherapeuten könnte Veränderungen bringen. Auch die Psychotherapie und weitere Versorgungsaufgaben bei psychischen Erkrankungen und psychisch relevanten Faktoren körperlicher Erkrankungen durch Allgemeinärzte, Psychiater und Psychosomatiker werden Adaptationen unterworfen werden, die zusätzlich von Fragen der ärztlichen Versorgung allgemein sowie des ärztlichen Nachwuchses im Besonderen beeinflusst werden. Welche Rolle für die psychosoziale Unterstützung werden nicht approbierte Heilhilfsberufe (z.B. Ergotherapeuten, Sozialpädagogen, Laien- und Krisenhelfer) sowie entsprechende Maßnahmen (Leistungen zur Teilhabe am Arbeitsleben) zukünftig spielen? Wie werden Krankenkassen und sonstige Kostenträger zukünftig die Wirtschaftlichkeits- und Qualitätssicherung organisieren (z.B. Antrags- und Gutachterverfahren, Fachkundeerfordernisse, Qualitätsnachweise auf der Ebene der einzelnen Therapie)? Auch die Forschung und Weiterentwicklung der VT wird auf diese gesellschaftlichen Entwicklungen reagieren müssen (z.B. Internettherapie) und zu klären haben, welche Behandlung bei welcher Störung maximal effizient und ökonomisch ist.
Die VT ist eine Therapierichtung, die für diese vielfältigen Herausforderungen bestens gerüstet ist. Deshalb ist parallel zu den aufgezeigten Entwicklungen eine Stärkung dieses Verfahrens zu erwarten. Unabhängig davon, wie die gesellschaftlichen und gesundheitspolitischen Entwicklungen verlaufen werden, wird die VT aufgrund ihrer Strukturiertheit, theoretischen Breite und soliden empirischen Basis mit ihrer vergleichsweise schnellen, ökonomischen und effizienten Arbeitsweise eine Schlüsselstellung innerhalb der heilkundlichen Psychotherapie einnehmen, die vom Wissenschaftlichen Beirat Psychotherapie und Gemeinsamen Bundesausschuss wissenschaftlich anerkannt ist. Die KVT ist und bleibt immer noch das Psychotherapieverfahren, dessen Wirksamkeit bei einer Vielzahl psychischer Störungen am besten belegt ist - trotz der Begeisterung für neue Therapieansätze.

VT ist auch ein Psychotherapieverfahren, das im Rahmen einer lediglich 3-jährigen Aus- oder Weiterbildung zu vermitteln ist. Dies ist dadurch bedingt, dass trotz der störungsspezifischen Differenziertheit die erforderlichen Grundkompetenzen oder «Basistechniken» der VT gut lehrbar sind (z.B. Verhaltensanalyse, Hausaufgaben, Aktivitätsaufbau, Analyse und Modifikation von Kognitionen, Exposition). Den Ausbildungskandidaten kann daher eine umfängliche Therapiekompetenz vermittelt werden, mit der sie eine große Vielzahl von Störungen und individuellen Problemlagen behandeln können. Gerade der Aspekt der Ausbildungskosten für ein spezifisches Verfahren wird und muss für eine Gesellschaft ebenfalls eine zunehmend wichtige Rolle spielen.

Die Auseinandersetzungen mit den Verfahren der sogenannten Dritten Welle können für die Weiterentwicklungen der VT eine wichtige Rolle spielen. Solche Auseinandersetzungen haben in der Historie der VT dazu geführt, dass sie daran wuchs und sich weiterentwickelte, und so möge es auch in Zukunft sein. Gleiches gilt für die Entwicklungen einer transdiagnostischen Psychotherapie, die stärker auf gemeinsame Mechanismen unterschiedlicher Störungen fokussiert als auf die spezifische Diagnose. Dazu kommt die Stärke der VT, (selbst-)kritisch zu analysieren, welcher Ansatz sich wann bewährt. So macht sich bezüglich der sogenannten Dritte-WelleVerfahren auch eine zunehmende Ernüchterung breit. Die Evidenzbasierung ist insgesamt noch unzureichend, und für die Mehrzahl der Störungsbilder lässt sich nicht schlussfolgern, dass diese Therapieverfahren wirksamer sind als die bisherige KVT. Je mehr Studien jenseits des Umfelds der charismatischen Gründerpersönlichkeiten der Dritte-Welle-Therapien durchgeführt werden, desto mehr nähern sich die Effektstärken der Dritte-Welle-Verfahren denen der traditionellen KVT an oder geraten sogar in den Bereich niedrigerer Effektstärken. Geringe Unterschiede lassen sich vor allem dann nachweisen, wenn störungsspezifische Effekte gemessen und selegierte Stichproben untersucht werden. Daraus lässt sich vor allem die Schlussfolgerung ziehen, dass ein störungsspezifisches Vorgehen effektiver ist als ein unspezifisches, jedoch braucht man hierfür nicht unbedingt ein neues Behandlungskonzept! Deshalb sollte die Begeisterung für die Verfahren der neuen Behandlungskonzepte nicht dazu führen, dass Ausbildungskandidaten die KVT als Ganzes nicht mehr gründlich und solide erlernen oder ihren Enthusiasmus dafür verlieren. 
Ein weiterer Trend könnte darin bestehen, die Person des Therapeuten näher zu beleuchten. Psychotherapie kann nur bedingt erlernt werden. Wie bei Musikalität braucht es auch hier «Talent». Dieser Umstand wurde bisher noch zu wenig berücksichtigt und beforscht.

Welche Voraussetzungen sind erforderlich, damit man bis zu 40 Jahre seines Lebens Psychotherapie ausüben kann? Welche Unterstützung kann hier die Profession anbieten?

Um von einer wissenschaftlich fundierten Psychotherapie sprechen zu können, bedarf es zwar zum einen kurz- und langfristiger Wirksamkeitsnachweise, aber zum anderen auch der Erfassung möglicher unerwünschter Therapieeffekte, beispielsweise ausgelöst durch unprofessionelles Verhalten des Therapeuten. Dieses bislang vernachlässigte Thema sollte künftig bereits in der Ausbildung behandelt werden.

Über die letzten Jahrzehnte und parallel zum Erfolg der VT sind jedoch auch Verluste zu verzeichnen. War die konzeptionelle Basis zu Beginn der VT noch klar definiert (Lernpsychologie, wissenschaftliche Fundierung), so wurden diese klaren Konturen, wie VT zu definieren ist, zunehmend verwischt. Eine Therapieform benötigt jedoch auch ein theoretisches Fundament und eine Grundlagenwissenschaft, auf die sie ihre Anwendungen aufbaut. Aus diesem Grund ist die enge Vernetzung mit den Konzepten und Erkenntnissen der Grundlagenpsychologie sowie der Neurowissenschaften wesentlich, damit VT nicht nur eine willkürliche Ansammlung verschiedener Behandlungsansätze ist, sondern diese auf einem gemeinsamen konzeptionellen Fundament basieren. Auch hier kann die VT die treibende Zugkraft sein, dass in Zukunft (gegebenenfalls auch gemeinsam mit anderen Therapieansätzen) auf gemeinsame theoretische Grundkonzepte aufgebaut wird und die verschiedenen Behandlungsansätze mit den Begriffen dieser Grundlagenwissenschaften beschrieben werden können. Es besteht damit die Chance, dass Therapieverfahren nicht in ihrer eigenen Sprache verhaftet bleiben, sondern man eine gemeinsame Sprache zur Beschreibung psychotherapeutischer Prozesse entwickelt.

Unsere Zeitschrift Verhaltenstherapie spielt vor diesem Hintergrund in absehbarer Zukunft weiterhin eine wichtige Rolle im Konzert der vielen anderen Psychotherapiezeitschriften. Sie wird Wissenschaftlern und Praktikern nachhaltig eine Plattform geben, um ihre Forschungsergebnisse und Praxiserfahrungen darzustellen. Sie wird über Neuentwicklungen in der VT berichten wie auch die Anwendung in der täglichen Routine und die psychotherapeutische Versorgung. Sie soll Lehrenden und Lernenden der VT als Einführung und Referenz dienen. Sie soll damit schließlich auch helfen, den Sozialversicherungen erkennbar zu machen, was VT ist und was nicht. Aber wir sind zuversichtlich: Keine Psychotherapieform scheint uns ähnlich gut für die Herausforderungen, die die Gegenwart und Zukunft bringen wird, gerüstet zu sein. 